\title{
Exploring the SPARK of Science with a New Light
}

Vivian Stojanoff ${ }^{a}$, Lisa Miller ${ }^{b}$ and Aleida Perez ${ }^{c}$

${ }^{a}$ National Synchrotron Light Source-II, Brookhaven National Laboratory, stojanof@bnl.gov

${ }^{b}$ National Synchrotron Light Source-II, Brookhaven National Laboratory, Imiller@bnl.gov

${ }^{c}$ Office of Educational Programs, Brookhaven National Laboratory, pereza@bnl.gov

The Student Partnerships for Advanced Research and Knowledge (SPARK) program at Brookhaven National Laboratory engages high school students and teachers in collaborative authentic research with scientists. At the National Synchrotron Light Source-II (NSLS-II), teams of student and teacher researchers leverage the tools at the NSLS-II to conduct original research. The SPARK program promotes collaborative research amongst schools in partnership with research institutions as a means of motivating students to consider careers in STEM, complementing their school curriculum content and enabling active contributions to science.

Currently twenty schools on Long Island participate in the program exploring scientific questions in crystallography and spectroscopy. Making use of a multimodal proposal submission model at NSLS II, the crystallography projects are concentrated on macromolecular crystallography and solution scattering beamlines while the spectroscopy projects make use of the powder diffraction beamlines at the NSLS-II. In this presentation we will discuss the SPARK program goals and impact, and will share some of the scientific contributions of the crystallography projects. 